\title{
Correction
}

\section{Correction: Bracci et al., "Representational Similarity of Body Parts in Human Occipitotemporal Cortex"}

In the article "Representational Similarity of Body Parts in Human Occipitotemporal Cortex" by Stefania Bracci, Alfonso Caramazza, and Marius V. Peelen, which appeared on pages 12977-12985 of the September 23, 2015 issue, the authors would like to correct an error in Figure 2, $B$ and $C$, fourth column ("cortical homunculus"), where the lower face and upper face icons have been interchanged. The correct order of the icons in the cortical homunculus model is the following: lower faces, upper faces, hands, arms, chests, waists, legs, feet. This correction does not affect the results, interpretation, or conclusions reported in the article. Figure 2 has been corrected on the on-line PDF version, and a corrected version displayed below.

A

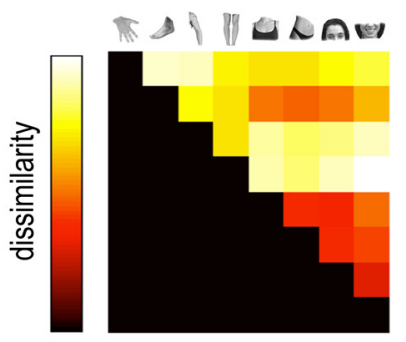

B

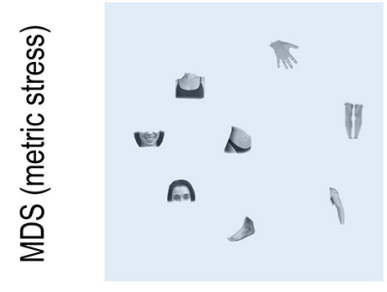

C

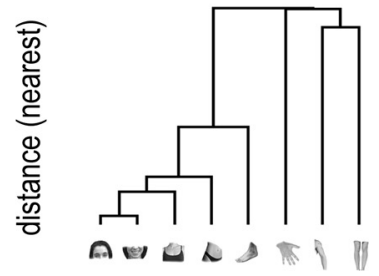

shape 1

(physical shape)
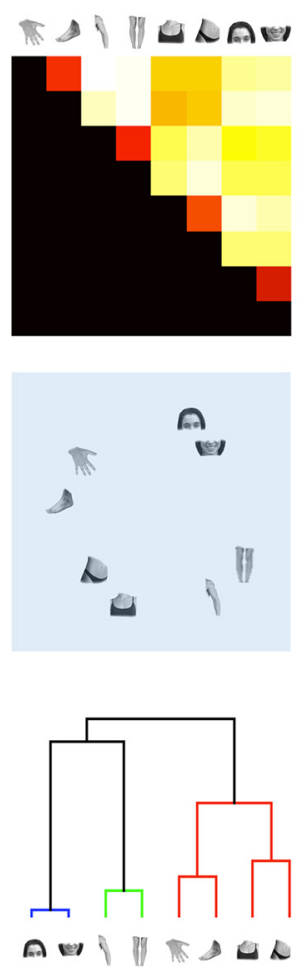

shape 2 (perceived shape)
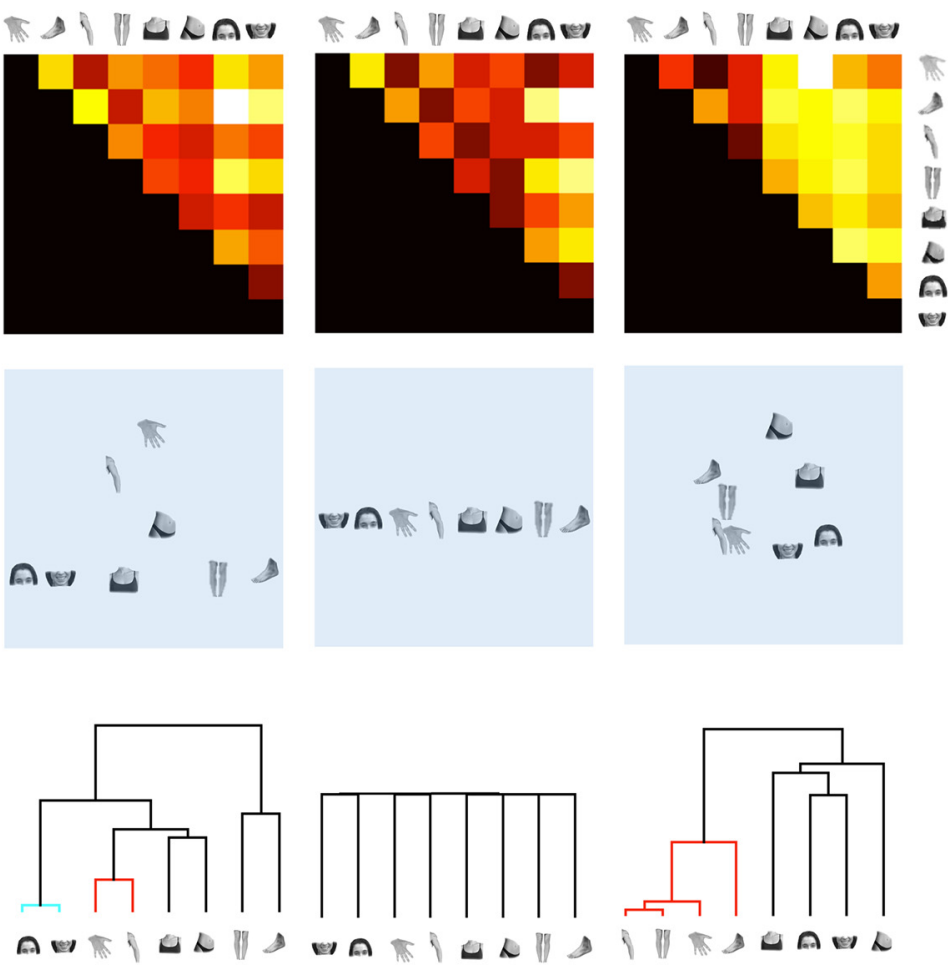

physical proximity

cortical homunculus

semantic

Figure 2. 